PROCEEDINGS OF THE

AMERICAN MATHEMATICAL SOCIETY

Volume 135, Number 6, June 2007, Pages 1619-1629

S 0002-9939(07)08738-2

Article electronically published on January 4, 2007

\title{
SOLUTIONS TO ARITHMETIC CONVOLUTION EQUATIONS
}

\author{
HELGE GLÖCKNER, LUTZ G. LUCHT, AND ŠTEFAN PORUBSKÝ
}

(Communicated by Jonathan M. Borwein)

\begin{abstract}
In the $\mathbb{C}$-algebra $\mathcal{A}$ of arithmetic functions $g: \mathbb{N} \rightarrow \mathbb{C}$, endowed with the usual pointwise linear operations and the Dirichlet convolution, let $g^{* k}$ denote the convolution power $g * \cdots * g$ with $k$ factors $g \in \mathcal{A}$. We investigate the solvability of polynomial equations of the form

$$
a_{d} * g^{* d}+a_{d-1} * g^{*(d-1)}+\cdots+a_{1} * g+a_{0}=0
$$

with fixed coefficients $a_{d}, a_{d-1}, \ldots, a_{1}, a_{0} \in \mathcal{A}$. In some cases the solutions have specific properties and can be determined explicitly. We show that the property of the coefficients to belong to convergent Dirichlet series transfers to those solutions $g \in \mathcal{A}$, whose values $g(1)$ are simple zeros of the polynomial $a_{d}(1) z^{d}+a_{d-1}(1) z^{d-1}+\cdots+a_{1}(1) z+a_{0}(1)$. We extend this to systems of convolution equations, which need not be of polynomial-type.
\end{abstract}

\section{INTRODUCTION}

The set of arithmetic functions $g: \mathbb{N} \rightarrow \mathbb{C}$ forms a $\mathbb{C}$-linear space $\mathcal{A}$ under pointwise addition and scalar multiplication. Null element is the null function 0. From the structural point of view, an appropriate multiplication is the Dirichlet convolution $(g, h) \in \mathcal{A} \times \mathcal{A} \mapsto g * h \in \mathcal{A}$ defined by

$$
(g * h)(n)=\sum_{n_{1} n_{2}=n} g\left(n_{1}\right) h\left(n_{2}\right) \quad(n \in \mathbb{N}),
$$

where the summation is taken over all pairs $\left(n_{1}, n_{2}\right) \in \mathbb{N}^{2}$ with $n_{1} n_{2}=n$. Convolution and pointwise addition make $\mathcal{A}$ into an integral domain with the identity element $\varepsilon$ defined by $\varepsilon(1)=1$ and $\varepsilon(n)=0$ for $1 \neq n \in \mathbb{N}$. We shall also tacitly use pointwise multiplication, but in the sequel we shall always consider $\mathcal{A}$ as a $\mathbb{C}$-algebra under linear operations and convolution. The multiplicative group of $\mathcal{A}$ with respect to the Dirichlet convolution is $\mathcal{A}^{*}=\{g \in \mathcal{A}: g(1) \neq 0\}$. For abbreviation we write $g^{* d}$ for the convolution $g * \cdots * g$ with $d$ factors $g \in \mathcal{A}$.

Received by the editors February 10, 2006.

2000 Mathematics Subject Classification. Primary 11A25, 46H30.

Key words and phrases. Arithmetic functions, Dirichlet convolution, polynomial equations, analytic equations, topological algebras, holomorphic functional calculus.

Research on this paper was begun while the second author visited the Academy of Sciences of the Czech Republic in Prague, partly supported by a travel grant of the Deutsche Forschungsgemeinschaft. He wishes to thank the Institute of Computer Science for their hospitality. The third author was supported by the Grant Agency of the Czech Republic, Grant\# 201/04/0381, and by the Institutional Research Plan AV0Z10300504. For some of the period during which this work was carried out he enjoyed the hospitality and support of the Clausthal University of Technology. 
The principle aim of this note is to deal with general polynomial-type convolution equations in one variable.

Theorem 1. For $d \in \mathbb{N}$ let $T: \mathcal{A} \rightarrow \mathcal{A}$ be defined by

$$
T g:=a_{d} * g^{* d}+a_{d-1} * g^{*(d-1)}+\cdots+a_{1} * g+a_{0}
$$

for $g \in \mathcal{A}$ with $a_{d}, a_{d-1}, \ldots, a_{1}, a_{0} \in \mathcal{A}$ and $a_{d} \neq 0$. If $z_{0}$ is a simple zero of the polynomial

$$
f(z)=a_{d}(1) z^{d}+a_{d-1}(1) z^{d-1}+\cdots+a_{1}(1) z+a_{0}(1),
$$

then there exists a uniquely determined solution $g \in \mathcal{A}$ to the convolution equation $T g=0$ satisfying $g(1)=z_{0}$. If $f(z)$ has no simple zeros, then $T g=0$ need not possess any solution. In any case $\mathrm{Tg}=0$ has at most d solutions.

The case of convolution equations of the form

$$
g^{* d}=a
$$

with given function $a \in \mathcal{A}, a \neq 0$, was studied repeatedly (see, e.g., Caroll and Gioia [2, Cohen 3], Dehaye [5], Haukkanen [9, 10, Porubský [14, Subbarao 17, and the papers quoted there; for polynomial convolution equations see also Rearick [15] and Laohakosol [13). In particular, it follows from Theorem 1 that equation (44) has exactly $d$ distinct solutions if $a \in \mathcal{A}^{*}$. The case $a \in \mathcal{A} \backslash \mathcal{A}^{*}$, i.e., $a(1)=0$, is delicate and will be discussed in Section 3 in the context of multiple zeros of $f(z)$.

There is no explicit formula for the solutions $g$ to $T g=0$, except for some specific cases. An important subgroup of $\mathcal{A}^{*}$ is the group $\mathcal{M}$ of multiplicative functions $g \in \mathcal{A}$, i.e., $g(1)=1$ and $g(m n)=g(m) g(n)$ for all coprime $m, n \in \mathbb{N}$. Let $\mathbb{P}$ denote the set of primes. Then each function $g \in \mathcal{M}$ is completely determined by its values $g\left(p^{k}\right)$ on the set $\mathbb{P}^{\star}=\left\{p^{k}: p \in \mathbb{P}, k \in \mathbb{N}\right\}$ of prime powers, and vice versa these values uniquely determine a function $g \in \mathcal{M}$. A function $g \in \mathcal{M}$ is said to be completely multiplicative if $g\left(p^{k}\right)=g^{k}(p):=(g(p))^{k}$ for all $p^{k} \in \mathbb{P}^{\star}$, it is called exponentially multiplicative if $g\left(p^{k}\right)=\frac{1}{k !} g^{k}(p)$ for all $p^{k} \in \mathbb{P}^{\star}$, and it is called 2-multiplicative (see Schwarz [16]) if $g\left(p^{k}\right)=0$ for all $p^{k} \in \mathbb{P}^{\star}$ with $k \geq 2$.

Caroll and Gioia 2], Subbarao [17, and Haukkanen [10] determined the solution $g \in \mathcal{M}$ to $g^{* 2}=a$ for specific functions $a \in \mathcal{M}$. In Section 3 we verify the following explicit formulas.

Theorem 2. Let $d \in \mathbb{N}$ and $a \in \mathcal{M}$. Then there is a unique solution $g \in \mathcal{M}$ to $g^{* d}=a$. In particular, $g \in \mathcal{M}$ is determined by its values at $p^{k} \in \mathbb{P}^{\star}$ as follows:

(a) $g\left(p^{k}\right)=\frac{1}{k !} \frac{a^{k}(p)}{d^{k}}$ if a is exponentially multiplicative,

(b) $g\left(p^{k}\right)=(-1)^{k}\left(\begin{array}{c}-\frac{1}{d} \\ k\end{array}\right) a^{k}(p)$ if a is completely multiplicative,

(c) $g\left(p^{k}\right)=\left(\begin{array}{c}\frac{1}{d} \\ k\end{array}\right) a^{k}(p)$ if a is 2-multiplicative.

The next theorem guarantees that under the conditions of Theorem 1 the solutions $g \in \mathcal{A}$ to $T g=0$, which correspond to simple zeros of $f(z)$, have absolutely convergent Dirichlet series in some open right half plane $H_{r}=\{s \in \mathbb{C}: \operatorname{Re} s>r\}$ with $r \geq 0$ of the complex plane if the coefficients $a_{j}$ have this property. By $\mathcal{A}_{r}$ we denote the complex Banach algebra of arithmetic functions $g \in \mathcal{A}$ with finite norm

$$
\|g\|_{r}:=\sum_{n=1}^{\infty}|g(n)| n^{-r} .
$$


Theorem 3. Let $a_{d}, a_{d-1}, \ldots, a_{1}, a_{0} \in \mathcal{A}_{\varrho}$ and $a_{d} \neq 0$ for some $\varrho \geq 0$. With the notations of Theorem 1, suppose that $g \in \mathcal{A}$ solves the convolution equation $T g=0$, where $g(1)$ is a simple zero of the polynomial $f(z)$. Then there exists some $r \geq \varrho$ such that $g \in \mathcal{A}_{r}$.

Theorem 3 is the special case

$$
m=1, \quad F: \mathbb{C}^{d+1} \times \mathbb{C} \rightarrow \mathbb{C}, \quad F\left(w_{0}, \ldots, w_{d}, z\right)=\sum_{j=0}^{d} w_{j} z^{j}
$$

of the following multi-dimensional version, which even applies to analytic equations. The theorem involves the functional transform $g \in \mathcal{A} \mapsto \widetilde{g} \in \widetilde{\mathcal{A}}$, which associates with $g$ the formal Dirichlet series

$$
\widetilde{g}(s)=\sum_{n=1}^{\infty} \frac{g(n)}{n^{s}} \quad(s \in \mathbb{C}) .
$$

The product of $\widetilde{g}, \widetilde{h} \in \widetilde{\mathcal{A}}$ is made into an element of $\widetilde{\mathcal{A}}$ by formally reordering the double series, i.e., $\widetilde{g}(s) \widetilde{h}(s)=(g * h)^{\sim}$. Indeed the $\mathbb{C}$-algebra $\mathcal{A}$ and the socalled Dirichlet algebra $\widetilde{\mathcal{A}}$ endowed with the usual linear operations and pointwise multiplication are isomorphic, and $\widetilde{g}(s)$ converges absolutely for all $s \in \bar{H}_{r}$, the closure of $H_{r}$, if and only if $g \in \mathcal{A}_{r}$.

Theorem 4. For open subsets $W \subseteq \mathbb{C}^{n}$ and $Z \subseteq \mathbb{C}^{m}$, let

$$
F: W \times Z \rightarrow \mathbb{C}^{m}, \quad(w, z) \mapsto F(w, z)
$$

be a holomorphic function and $\left(w_{0}, z_{0}\right) \in W \times Z$ with $F\left(w_{0}, z_{0}\right)=0$. Let $a_{1}, \ldots, a_{n}$ $\in \mathcal{A}$ be elements such that $\left(a_{1}(1), \ldots, a_{n}(1)\right)=w_{0}$. If the differential $\partial_{z} F\left(w_{0}, z\right)$ at $z=z_{0}$ is in $\mathrm{GL}_{m}(\mathbb{C})$, then there exists a unique $m$-tuple $\left(g_{1}, \ldots, g_{m}\right) \in \mathcal{A}^{m}$ such that

$$
\begin{aligned}
\left(g_{1}(1), \ldots, g_{m}(1)\right) & =z_{0} \quad \text { and } \\
F\left(a_{1}, \ldots, a_{n}, g_{1}, \ldots, g_{m}\right) & =0 .
\end{aligned}
$$

If, furthermore, $a_{1}, \ldots, a_{n} \in \mathcal{A}_{\varrho}$ for some $\varrho \geq 0$, then there is an $r \geq \varrho$ such that $g_{1}, \ldots, g_{m} \in \mathcal{A}_{r},\left(\widetilde{a}_{1}(s), \ldots, \widetilde{a}_{n}(s), \widetilde{g}_{1}(s), \ldots, \widetilde{g}_{m}(s)\right) \in W \times Z$, and

$$
F\left(\widetilde{a}_{1}(s), \ldots, \widetilde{a}_{n}(s), \widetilde{g}_{1}(s), \ldots, \widetilde{g}_{m}(s)\right)=0 \quad \text { for all } s \in \bar{H}_{r} .
$$

In this case, (6) and (8) also uniquely determine $\left(g_{1}, \ldots, g_{m}\right) \in \mathcal{A}_{r}^{m}$.

Here, the left-hand side of (7) is obtained by inserting algebra elements into the Taylor expansion of $F$ around $\left(w_{0}, z_{0}\right)$.

Section 4 provides a proof of Theorem 4 , which is based on techniques from the theory of commutative topological algebras.

\section{Proof of Theorem 1 And comments}

For the proof of Theorem 1 we rewrite $T g=0$ as an infinite system of equations

$$
\sum_{\ell m=n}\left(a_{d}(\ell) g^{* d}(m)+a_{d-1}(\ell) g^{*(d-1)}(m)+\cdots+a_{0}(\ell) \varepsilon(m)\right)=0
$$

for $n \in \mathbb{N}$. It follows from (9) at $n=1$ that $f(g(1))=0$. Each simple root, say $z_{0}$, of the equation $f(z)=0$ can be used as a starting value for $g(1)=z_{0}$ in the following procedure. 
By evaluating $h_{j}:=a_{j} * g^{* j}$ at $n \in \mathbb{N}, n \neq 1$, and separating all terms which contain $g(n)$, we obtain for $j \in \mathbb{N}$ that

$$
h_{j}(n)=j a_{j}(1) g^{j-1}(1) \cdot g(n)+\sum_{\substack{\ell n_{1} \cdots n_{j}=n \\ n_{1}, \ldots, n_{j}<n}} a_{j}(\ell) g\left(n_{1}\right) \cdots g\left(n_{j}\right) .
$$

Here the coefficient of $g(n)$ equals the value of the derivative $\left(a_{j}(1) z^{j}\right)^{\prime}$ at $z=g(1)$, while the remaining sum contains values $g(m)$ with $m<n$ only. Therefore (9) takes the form

$$
f^{\prime}(g(1)) \cdot g(n)=-\sum_{0 \leq j \leq d} \sum_{\substack{\ell n_{1} \cdots n_{j}=n \\ n_{1}, \ldots, n_{j}<n}} a_{j}(\ell) g\left(n_{1}\right) \cdots g\left(n_{j}\right) \quad(n>1) .
$$

Due to the choice of $g(1)=z_{0}$ we have $f^{\prime}(g(1)) \neq 0$ so that (10) represents a recursion formula, which uniquely determines an arithmetic function $g \in \mathcal{A}$.

Let $T g=g * g-a$ with $a \in \mathcal{A}$ satisfying $a(1)=0 \neq a(p)$ for some prime $p$, say. Then $f(z)=z^{2}$ and $f^{\prime}(z)=2 z$ both vanish at $z=0$. The convolution equation $T g=0$ yields $T g(1)=0$ at $g(1)=0$ only. Since $T g(p)=2 g(1) g(p)-a(p)=$ $-a(p) \neq 0$, there exists no solution to $T g=0$.

Since $\mathcal{A}$ is an integral domain, the polynomial $T g$ of degree $d$ has at most $d$ zeros $g \in \mathcal{A}$.

As an immediate consequence of Theorem 1 we obtain

Corollary 1. If $f(z)$ satisfies $\operatorname{deg} f=d$ and all zeros of $f(z)$ are simple, then, with the $d$ distinct solutions $g_{1}, \ldots, g_{d} \in \mathcal{A}$ to the convolution equation $T g=0$, we have

$$
T g=a_{d} *\left(g-g_{1}\right) * \cdots *\left(g-g_{d}\right) .
$$

Remark 1. Theorem 1 covers the case of convolution polynomials

$$
T g:=\alpha_{d} g^{* d}+\alpha_{d-1} g^{*(d-1)}+\cdots+\alpha_{1} g+\alpha_{0} \varepsilon=0
$$

with constant coefficients $\alpha_{j} \in \mathbb{C}, \alpha_{d} \neq 0$, and the identity element $\varepsilon \in \mathcal{A}$, which is seen by setting $a_{j}=\alpha_{j} \varepsilon \in \mathcal{A}$ for $0 \leq j \leq d$.

Corollary 2. Let $a \in \mathcal{A}^{*}$ and $d \in \mathbb{N}$. If $g_{0} \in \mathcal{A}$ is one solution to $g^{* d}=a$, then all solutions are given by $\omega_{j} g_{0}$, where $\omega_{j}$ runs over all dth complex roots of unity.

Proof. The assumptions of Theorem 1 are satisfied for $f(z)=z^{d}-a(1)$ since $a(1) \neq 0$. The assertion now follows by inserting the $d$ distinct sequences $\omega_{j} g_{0}$ for $j=0,1, \ldots, d-1$ into $T g=g^{* d}-a$.

\section{Multiple Zeros}

As the case of multiple zeros of $f(z)$ has led to several incorrect statements in the literature, we shall briefly discuss it. Following Knopfmacher [12, we first define the order $\langle g\rangle$ of a function $g \in \mathcal{A}$ by

$$
\langle g\rangle= \begin{cases}\min \{n \in \mathbb{N}: g(n) \neq 0\} & \text { if } 0 \neq g \in \mathcal{A}, \\ \infty & \text { if } g=0\end{cases}
$$

Then $\langle g\rangle=1$ if and only if $g \in \mathcal{A}^{*}$, and $\langle g * h\rangle=\langle g\rangle \cdot\langle h\rangle$ for all $g, h \in \mathcal{A}$. 
Remark 2. Theorem 5 of Subbarao [17] states that the convolution equation $g^{* d}=a$ has exactly $d$ solutions $g \in \mathcal{A}$ if $0 \neq a \in \mathcal{A}$. This is incorrect for $d>1$, since any solution $g$ satisfies $\langle g\rangle^{d}=\langle a\rangle$ so that necessarily $\langle a\rangle$ is the $d$ th power of some positive integer. Therefore, e.g., $g * g=a$ has no solution if $a(1)=0$ and $a(2) \neq 0$.

Remark 3. Theorem 1 of Haukkanen 9] states that the order condition $\langle a\rangle=m^{d}$ with some $m \in \mathbb{N}$ from Remark 2 guarantees the solvability of $g^{* d}=a$. But this does not suffice, in general. E.g., if $d>1$ and $a(1)=0$, then every solution $g$ satisfies $0=a(1)=g^{d}(1)$ so that $g(1)=0$ and $a(p)=d g^{d-1}(1) g(p)=0$ for all primes $p$. Hence $g * g=a$ has no solution if $a(1)=0$ and $a(p) \neq 0$ for some $p \in \mathbb{P}$ with $p>\langle a\rangle$.

Remark 4. Theorem 3 of Haukkanen 9 states that the linear convolution equation $a * g=b$ is solvable if the divisibility condition $\langle a\rangle \mid\langle b\rangle$ is satisfied. In general, this divisibility condition does not suffice. E.g., if $a(1)=b(1)=0$, then $b(p)=$ $a(1) g(p)+a(p) g(1)=a(p) g(1)$ for all $p \in \mathbb{P}$. Therefore $a * g=b$ has no solution if $a(1)=b(1)=0$ and $a(p)=0 \neq b(p)$ for some prime $p$ with $p>\langle a\rangle$.

As the preceding remarks show, the convolution equation need not have any solution if $g(1)$ is a multiple zero of $f(z)$. In fact, if $f(g(1))=f^{\prime}(g(1))=0$, then the coefficient functions $a_{j} \in \mathcal{A}$ of $T g$ in (2) underlie severe restrictions. We exemplarily derive some conditions, which are necessary for the existence of solutions $g \in \mathcal{A}$ to $T g=0$.

For $m \in \mathbb{N}$ we introduce the polynomials $f_{m}(z) \in \mathbb{C}[z]$ by

$$
f_{m}(z)=a_{d}(m) z^{d}+\cdots+a_{1}(m) z+a_{0}(m) .
$$

Obviously $f(z)=f_{1}(z)$. Suppose now that $f_{1}\left(z_{0}\right)=f_{1}^{\prime}\left(z_{0}\right)=0$ and $g$ is a solution to $T g=0$ satisfying $g(1)=z_{0}$.

i) For $p \in \mathbb{P}$ we find

$$
\begin{aligned}
T g(p) & =\sum_{0 \leq j \leq d} a_{j}(p) g^{j}(1)+g(p) \sum_{1 \leq j \leq d} a_{j}(1) j g^{j-1}(1) \\
& =f_{p}\left(z_{0}\right)+g(p) f_{1}^{\prime}\left(z_{0}\right) .
\end{aligned}
$$

It follows from $f^{\prime}\left(z_{0}\right)=0$ that $z_{0}$ is a zero of $f_{p}(z)$ for all $p \in \mathbb{P}$.

ii) In the same way we obtain for $p \in \mathbb{P}$

$$
T g\left(p^{2}\right)=f_{p^{2}}\left(z_{0}\right)+g(p) f_{p}^{\prime}\left(z_{0}\right)+g\left(p^{2}\right) f_{1}^{\prime}\left(z_{0}\right)+\frac{1}{2} g^{2}(p) f_{1}^{\prime \prime}\left(z_{0}\right),
$$

and $f^{\prime}\left(z_{0}\right)=0$ implies that $z_{0}$ is a zero of $f_{p^{2}}(z)+g(p) f_{p}^{\prime}(z)+\frac{1}{2} g^{2}(p) f_{1}^{\prime \prime}(z)$.

iii) For distinct $p, q \in \mathbb{P}$ we have

$$
T g(p q)=f_{p q}\left(z_{0}\right)+g(q) f_{p}^{\prime}\left(z_{0}\right)+g(p) f_{q}^{\prime}\left(z_{0}\right)+g(p q) f_{1}^{\prime}\left(z_{0}\right)+g(p) g(q) f_{1}^{\prime \prime}\left(z_{0}\right) .
$$

Now $f^{\prime}\left(z_{0}\right)=0$ implies that $z_{0}$ is a zero of $f_{p q}(z)+g(p) f_{q}^{\prime}(z)+g(q) f_{p}^{\prime}(z)+$ $g(p) g(q) f_{1}^{\prime \prime}(z)$ for all distinct $p, q \in \mathbb{P}$.

For instance, let $T g=g * g-a$ with $a \in \mathcal{A}, a(1)=0$. Then $f_{q}(z)=z^{2}-a(q)$ for all $q \in \mathbb{N}$, particularly $z_{0}=0$ is a double zero of $f_{1}(z)$. We get $f_{q}^{\prime}(z)=2 z$ and $f_{q}^{\prime \prime}(z)=2$ for all $q \in \mathbb{N}$ so that $f_{q}(0)=-a(q), f_{q}^{\prime}(0)=0, f_{q}^{\prime \prime}(0)=2$. From ii) and iii) we infer that $-a\left(p^{2}\right)-g^{2}(p)=0,-a\left(q^{2}\right)-g^{2}(q)=0$, and $-a(p q)+2 g(p) g(q)=0$ for distinct primes $p, q$. It follows that the values of $a$ are interrelated by $a^{2}(p q)=a\left(p^{2}\right) a\left(q^{2}\right)$ for all $p, q \in \mathbb{P}, p \neq q$, if a solution $g$ to $T g=0$ exists. 
Observe that the above examples i), ii), and iii) can be extended to products of more than two prime factors. It seems to be difficult to provide fairly general sufficient conditions on the coefficients $a_{j}$ of the convolution polynomial $\mathrm{Tg}$ characterizing the solvability of $T g=0$ in the case of multiple zeros $z_{0}=g(1)$ of $f(z)$.

\section{Proof of Theorem 2 And comments}

The formal Dirichlet series $\widetilde{g}(s)$ of $g \in \mathcal{M}$ has a formal Euler product representation

$$
\widetilde{g}(s)=\prod_{p \in \mathbb{P}} \widetilde{g}_{p}(s) \quad(s \in \mathbb{C}),
$$

where the factor

$$
\widetilde{g}_{p}(s)=1+\frac{g(p)}{p^{s}}+\frac{g\left(p^{2}\right)}{p^{2 s}}+\cdots
$$

is the formal Dirichlet series of the $p$-fibre $g_{p} \in \mathcal{M}$ of $g$ defined by ${ }^{1}$

$$
g_{p}(n)=\left\{\begin{array}{ll}
g(n) & \text { if } n=p^{k} \text { for some } k \in \mathbb{N}_{0}=\mathbb{N} \cup\{0\} \\
0 & \text { otherwise }
\end{array} \quad(n \in \mathbb{N}) .\right.
$$

Note that, in the sense of convergence, (11) holds for all $s \in \bar{H}_{r}$ if $g \in \mathcal{A}_{r}$.

Proof of Theorem 2, Due to Corollary 2 there exists a uniquely determined solution $g \in \mathcal{A}$ to $g^{* d}=a$ such that $g(1)=1$. Let the function $h \in \mathcal{M}$ be defined by $h\left(p^{k}\right)=g\left(p^{k}\right)$ for all prime powers $p^{k} \in \mathbb{P}^{\star}$ and by multiplicative continuation from $\mathbb{P}^{\star}$ to $\mathbb{N}$. Since $\mathcal{M}$ is closed under the convolution, $h$ is a multiplicative solution to $g^{* d}=a$ with $h(1)=1$. Hence $g=h \in \mathcal{M}$.

The strategy for verifying the statements (a) to (c) consists in reducing the global convolution equation $g^{* d}=a$ to its local versions $g_{p}^{* d}=a_{p}$ for all $p$-fibres and switching to the associated formal Euler product factors $\widetilde{g}_{p}^{d}(s)=\widetilde{a}_{p}(s)$ or equivalently to $\widetilde{g}_{p}(s)=\left(\widetilde{a}_{p}(s)\right)^{1 / d} \cdot{ }^{2}$ With $z=p^{-s}$ this takes the form (compare Subbarao [17, Section 3])

$$
1+g(p) z+g\left(p^{2}\right) z^{2}+\cdots=\left(1+a(p) z+a\left(p^{2}\right) z^{2}+\cdots\right)^{1 / d}
$$

If the right-hand side coincides with a power series expansion of some known analytic function, then the values of $g \in \mathcal{M}$ at prime powers $p^{k} \in \mathbb{P}^{\star}$ result from comparing coefficients.

(a) It follows from $a\left(p^{k}\right)=\frac{a^{k}(p)}{k !}$ that $1+a(p) z+a\left(p^{2}\right) z^{2}+\cdots=\exp (a(p) z)$. From (13) and the exponential series we see that

$$
\sum_{k=0}^{\infty} g\left(p^{k}\right) z^{k}=\exp \left(\frac{a(p)}{d} z\right)=\sum_{k=0}^{\infty} \frac{a^{k}(p)}{k ! d^{k}} z^{k}
$$

from which the stated formula follows by comparison.

(b) Now $a\left(p^{k}\right)=a^{k}(p)$ implies $1+a(p) z+a\left(p^{2}\right) z^{2}+\cdots=(1-a(p) z)^{-1}$. Therefore, via (13) and the binomial series, we have

$$
\sum_{k=0}^{\infty} g\left(p^{k}\right) z^{k}=(1-a(p) z)^{-\frac{1}{d}}=\sum_{k=0}^{\infty}(-1)^{k}\left(\begin{array}{c}
-\frac{1}{d} \\
k
\end{array}\right) a^{k}(p) z^{k}
$$

\footnotetext{
${ }^{1}$ Notation used for the $p$-fibre of $g$ within this section only.

${ }^{2} z^{1 / d}$ denotes the branch of the $d$ th root of $z$, which is positive for positive $z$.
} 
which yields the stated formula.

(c) From $a\left(p^{k}\right)=0$ for $k \geq 2$ we obtain $1+a(p) z+a\left(p^{2}\right) z^{2}+\cdots=1+a(p) z$. This gives

$$
\sum_{k=0}^{\infty} g\left(p^{k}\right) z^{k}=(1+a(p) z)^{\frac{1}{d}}=\sum_{k=0}^{\infty}\left(\begin{array}{l}
\frac{1}{d} \\
k
\end{array}\right) a^{k}(p) z^{k}
$$

and the stated formula appears.

For rational exponents $q=\frac{d}{m} \in \mathbb{Q}$ with coprime integers $d \in \mathbb{N}, m \in \mathbb{Z}$ and functions $a \in \mathcal{A}^{*}$, we may define $g^{* q}=a$ by $g^{* d}=a^{* m}$. The proof of Theorem 2 then easily transfers to

Corollary 3. With $q \in \mathbb{Q}$ instead of $d \in \mathbb{N}$, all assertions of Theorem 2 remain valid for convolution equations of the form $g^{* q}=a$.

\section{Proof of Theorem 4}

We find it convenient to deduce Theorem 4 from general facts concerning analytic equations in topological algebras. Recall that a complex topological algebra is an algebra $A$, equipped with a locally convex vector topology making the bilinear algebra multiplication $A \times A \rightarrow A$ a continuous map. It is called complete if the underlying locally convex space is complete. A continuous inverse algebra is a unital, associative complex topological algebra $A$ whose group of units $A^{*}$ is open in $A$ and whose inversion map $A^{*} \rightarrow A, a \mapsto a^{-1}$ is continuous (see Biller [1], Glöckner [8], and Waelbroeck [18]). The spectrum of a commutative continuous inverse algebra $A$ is the set $\widehat{A}$ of all algebra homomorphisms $\xi: A \rightarrow \mathbb{C}$. It is known that $\xi \mapsto \operatorname{ker} \xi$ is a bijection from $\widehat{A}$ onto the set of all maximal (proper) ideals of $A$ (Biller [1, Lemma 1.5]). The spectrum of an element $a \in A$ is defined as $\sigma(a):=\left\{s \in \mathbb{C}: s-a \notin A^{*}\right\}$, and by Biller [1, Theorem $\left.1.7(\mathrm{a})\right]$, it coincides with the set $\{\xi(a): \xi \in \widehat{A}\}$. Our proof of Theorem 4 rests on the following special case of Biller [1, Theorem 7.2], applied to algebras whose spectrum is a singleton.

Lemma 1. Let $A$ be a complete, commutative continuous inverse algebra whose spectrum is a singleton, $\widehat{A}=\{\xi\}$. Let $W \subseteq \mathbb{C}^{n}$ and $Z \subseteq \mathbb{C}^{m}$ be open sets and let $F: W \times Z \rightarrow \mathbb{C}^{m}$ be a holomorphic function. Suppose that $w_{0} \in W, z_{0} \in Z$ such that $F\left(w_{0}, z_{0}\right)=0$ and $\left.\partial_{z} F\left(w_{0}, z\right)\right|_{z=z_{0}} \in \mathrm{GL}_{m}(\mathbb{C})$. Then, for each $\left(a_{1}, \ldots, a_{n}\right) \in A^{n}$ satisfying $\left(\xi\left(a_{1}\right), \ldots, \xi\left(a_{n}\right)\right)=w_{0}$, there exists a unique $\left(g_{1}, \ldots, g_{m}\right) \in A^{m}$ such that $\left(\xi\left(g_{1}\right), \ldots, \xi\left(g_{m}\right)\right)=z_{0}$ and

$$
F\left(a_{1}, \ldots, a_{n}, g_{1}, \ldots, g_{m}\right)=0 .
$$

Remark 5. The left-hand side of (14) is defined componentwise using multi-variable holomorphic functional calculus. We recall: If $f: \mathbb{C}^{n} \supseteq U \rightarrow \mathbb{C}$ is a holomorphic function, $A$ a complete, commutative continuous inverse algebra and $a=$ $\left(a_{1}, \ldots, a_{n}\right) \in A^{n}$ such that $\sigma\left(a_{1}\right) \times \cdots \times \sigma\left(a_{n}\right) \subseteq U$, then the holomorphic functional calculus defines an element $F(a) \in A$ (see Biller [1] and the references therein). Hence, for $\widehat{A}=\{\xi\}$ a singleton, $F(a)$ can be formed for each $a \in A^{n}$ such that $x_{0}:=\left(\xi\left(a_{1}\right), \ldots, \xi\left(a_{n}\right)\right) \in U$. If $x_{0}$ is contained in a polycylinder $P \subseteq U$ around some element $u_{0} \in U$, and $F(u)=\sum_{\alpha \in \mathbb{N}_{0}^{n}} b_{\alpha}\left(u-u_{0}\right)^{\alpha}$ is the Taylor expansion of $F$ around $u_{0}$ (using multi-index notation), then $F(a)=\sum_{\alpha \in \mathbb{N}_{0}^{n}} b_{\alpha}\left(a-u_{0}\right)^{\alpha}$ in $A$. 
We shall apply the lemma to two algebras. First, to the algebra $\mathcal{A}=\mathbb{C}^{\mathbb{N}}$ of arithmetic functions, equipped with the product topology, which makes $\mathcal{A}$ a Fréchet space. Second, to

$$
\mathcal{A}_{\infty}:=\bigcup_{\varrho \geq 0} \mathcal{A}_{\varrho}
$$

which inherits a commutative, associative complex algebra structure from the algebras $\mathcal{A}_{\varrho}$. We give $\mathcal{A}_{\infty}$ the locally convex vector topology making it the locally convex direct limit

$$
\mathcal{A}_{\infty}=\lim _{\varrho} \mathcal{A}_{\varrho}=\underline{\lim }_{n \in \mathbb{N}} \mathcal{A}_{n} .
$$

The elements of $\mathcal{A}_{\infty}$ can be identified with germs around $+\infty$ of Dirichlet series that converge absolutely on some right half-plane.

Lemma 2. $\mathcal{A}$ is a commutative continuous inverse algebra whose spectrum is a singleton, namely $\widehat{\mathcal{A}}=\{\xi\}$ with $\xi: \mathcal{A}_{\infty} \rightarrow \mathbb{C}, f \mapsto f(1)$. Furthermore, $\mathcal{A}$ is a Fréchet space.

Proof. The point evaluation $\xi: f \mapsto f(1)$ is a continuous homomorphism $\mathcal{A} \rightarrow \mathbb{C}$. Let $\mathfrak{m}$ be its kernel. Then $\mathcal{A} \backslash \mathfrak{m}=\{f \in \mathcal{A}: f(1) \neq 0\}$ is the set $\mathcal{A}^{*}$ of invertible elements which entails that $\mathfrak{m}$ is the only maximal ideal of $\mathcal{A}$ and hence $\widehat{\mathcal{A}}=\{\xi\}$. Since $(f * g)(n)=\sum_{n_{1} n_{2}=n} f\left(n_{1}\right) g\left(n_{2}\right)$ is a polynomial in finitely many components of $f$ and $g$, it is continuous in $(f, g) \in \mathcal{A} \times \mathcal{A}$, whence convolution is continuous as a map $\mathcal{A} \times \mathcal{A} \rightarrow \mathcal{A}$. Given $f \in \mathcal{A}^{*}$, the component $f^{-1}(n)$ is a rational function in finitely many components of $f$ and hence continuous in $f$, entailing that the inversion map $\mathcal{A}^{*} \rightarrow \mathcal{A}$ is continuous.

Lemma 3. The following hold:

(a) For each $r \geq 0$, the map $\psi_{r}: \mathcal{A}_{0} \rightarrow \mathcal{A}_{r}, \psi_{r}(f)(n):=n^{r} f(n)$ is an isomorphism of Banach algebras.

(b) If $f \in \mathcal{A}_{0}$ and $g:=\psi_{r}(f)$, then $\widetilde{f}(s)=\widetilde{g}(s+r)$ for each $s \in \bar{H}_{0}$.

(c) For each $f \in \mathcal{A}_{r}$, its spectrum is the closure of $\widetilde{f}\left(\bar{H}_{r}\right)$ in $\mathbb{C}$.

(d) If $f \in \mathcal{A}_{\varrho}$ and $r \geq \varrho$, let $\sigma_{r}(f)$ be the spectrum of $f$ as an element of $\mathcal{A}_{r}$. Then $\sigma_{r}(f) \rightarrow f(1)$ as $r \rightarrow \infty$.

Proof. (a) and (b) are clear. If $r=0$, then (c) is covered by Hewitt and Williamson [11, Theorem 1]. Combining this special case with (a) and (b), the general case follows. Since $\widetilde{f}(s) \rightarrow f(1)$ as $\operatorname{Re}(s) \rightarrow \infty$, given $\varepsilon>0$ we find $r_{0} \geq \varrho$ such that $|\widetilde{f}(s)-f(1)| \leq \varepsilon$ for all $s \in H_{r_{0}}$. Using (c), we deduce that $\sigma_{r}(f)$ is contained in the closed disk of radius $\varepsilon$ around $f(1)$, for all $r \geq r_{0}$.

Lemma 4. $\mathcal{A}_{\infty}$ is a commutative continuous inverse algebra whose spectrum is a singleton, namely $\widehat{\mathcal{A}}_{\infty}=\{\eta\}$ with $\eta: \mathcal{A}_{\infty} \rightarrow \mathbb{C}, f \mapsto f(1)$. Also, $\mathcal{A}_{\infty}$ is a Silva space and hence complete.

Proof. If $0 \leq \varrho<r$, then the weights $\left(n^{-\varrho}\right)_{n \in \mathbb{N}}$ and $\left(n^{-r}\right)_{n \in \mathbb{N}}$ satisfy $\lim \left(\frac{n^{-r}}{n^{-\varrho}}\right)=0$, entailing that the inclusion map $\mathcal{A}_{\varrho} \rightarrow \mathcal{A}_{r}$ is a compact operator (see Floret and Wloka [7, $\S 19$, pp. 90-91]). Hence $\mathcal{A}_{\infty}=\lim _{n \in \mathbb{N}} \mathcal{A}_{n}$ is a Silva space (an (FS)-space in the terminology of Floret [6]) and thus complete by Floret [․ p. 170, Satz]. Like any direct limit of a direct sequence of normed algebras, $\mathcal{A}_{\infty}$ is a locally convex topological algebra which is locally m-convex (a subalgebra of a projective limit of normed algebras), by Dierolf and Wengenroth [4, Theorem 1]. This entails that 
the inversion map $\mathcal{A}_{\infty}^{*} \rightarrow \mathcal{A}_{\infty}$ is continuous. To see that $\mathcal{A}_{\infty}^{*}$ is open, we first note that the linear map $\eta$ is continuous because $\mathcal{A}_{\infty}$ is the locally convex direct limit of the spaces $\mathcal{A}_{\varrho}$ and $\left.\eta\right|_{\mathcal{A}_{\varrho}}$ is continuous for each $\varrho \geq 0$. Hence $\mathfrak{m}:=\operatorname{ker} \eta$ is closed, and thus $\mathcal{A}_{\infty}$ will be a continuous inverse algebra if we can show that $\mathcal{A}_{\infty}^{*}=A \backslash \mathfrak{m}$. Since $\mathfrak{m}$ is a proper ideal, we have $\mathcal{A}_{\infty}^{*} \subseteq A \backslash \mathfrak{m}$. To prove the converse inclusion, let $f \in \mathcal{A}_{\infty}$ with $\eta(f)=f(1) \neq 0$, where $f \in \mathcal{A}_{\varrho}$, say. Then $\lim _{\operatorname{Re}(s) \rightarrow \infty} \tilde{f}(s)=f(1) \neq 0$, whence there is an $r \geq \varrho$ such that the closure of $\widetilde{f}\left(\bar{H}_{r}\right)$ in $\mathbb{C}$ is contained in $\mathbb{C}^{*}$. Hence $\sigma(f) \subseteq \mathbb{C}^{*}$ holds for $f$ as an element of $\mathcal{A}_{r}$, by Lemma 3 . Therefore $f \in \mathcal{A}_{r}^{*}$ and hence also $f \in \mathcal{A}_{\infty}^{*}$. Thus $\mathcal{A}_{\infty}^{*}$ is open, and also we infer (as in the proof of Lemma 2) that $\operatorname{ker} \eta$ is the unique maximal ideal of $\mathcal{A}_{\infty}$ and thus $\widehat{\mathcal{A}}_{\infty}=\{\eta\}$.

Proof of Theorem 4. We proceed in steps.

Step 1. By Lemma 2, $\mathcal{A}$ is a complete, commutative continuous inverse algebra with spectrum $\{\xi\}$. Since $\left(\xi\left(a_{1}\right), \ldots, \xi\left(a_{n}\right)\right)=\left(a_{1}(1), \ldots, a_{n}(1)\right)=w_{0}$, Lemma 1 shows the existence and uniqueness of $\left(g_{1}, \ldots, g_{m}\right) \in \mathcal{A}^{m}$ such that conditions (6) and (7) of Theorem 4 are satisfied.

Step 2. Likewise, if $a_{1}, \ldots, a_{n} \in \mathcal{A}_{\varrho}$ for some $\varrho \geq 0$, then Lemma 1 and Lemma 4 provide a uniquely determined $m$-tuple $\left(g_{1}, \ldots, g_{m}\right) \in \mathcal{A}_{\infty}^{m}$ such that (6) and (7) hold. The elements $\left(g_{1}, \ldots, g_{m}\right) \in \mathcal{A}_{\infty}^{m}$ coincide with the corresponding elements of $\mathcal{A}$ obtained in Step 1 . To see this, note that the inclusion map $\lambda: \mathcal{A}_{\infty} \rightarrow \mathcal{A}$ is continuous linear (as its restriction to each $\mathcal{A}_{r}$ is continuous linear) and multiplicative, whence

$$
0=\lambda\left(F\left(a_{1}, \ldots, a_{n}, g_{1}, \ldots, g_{m}\right)\right)=F\left(\lambda\left(a_{1}\right), \ldots, \lambda\left(a_{n}\right), \lambda\left(g_{1}\right), \ldots, \lambda\left(g_{m}\right)\right)
$$

due to the naturality of holomorphic functional calculus (see Biller [1, Theorem 3.9]). Now the uniqueness assertion from Step 1 applies.

Step 3. There is an $r \geq \varrho$ such that $g_{1}, \ldots, g_{m} \in \mathcal{A}_{r}$. By Lemma 3(d), after increasing $r$ if necessary, we may assume that $\sigma_{r}\left(a_{1}\right) \times \cdots \times \sigma_{r}\left(a_{n}\right) \subseteq W$ and $\sigma_{r}\left(g_{1}\right) \times \cdots \times \sigma_{r}\left(g_{m}\right) \subseteq Z$. Thus $b:=F\left(a_{1}, \ldots, a_{n}, g_{1}, \ldots, g_{m}\right)$ can be defined in the commutative Banach algebra $\mathcal{A}_{r}$ using holomorphic functional calculus. The inclusion map $\mathcal{A}_{r} \rightarrow \mathcal{A}_{\infty}$ being a continuous algebra homomorphism, we deduce as above that $b$ coincides with $F\left(a_{1}, \ldots, a_{n}, g_{1}, \ldots, g_{m}\right)$ calculated in $\mathcal{A}_{\infty}$, which is 0 . Thus $b=0$ and hence (8) holds. In fact, for each $s \in \bar{H}_{r}$, the map

$$
\widehat{s}: \mathcal{A}_{r} \rightarrow \mathbb{C}, \quad \widehat{s}(f):=\tilde{f}(s)
$$

is an algebra homomorphism, and thus

$$
\begin{aligned}
0=\widehat{s}(b) & =F\left(\widehat{s}\left(a_{1}\right), \ldots, \widehat{s}\left(a_{n}\right), \widehat{s}\left(g_{1}\right), \ldots, \widehat{s}\left(g_{m}\right)\right) \\
& =F\left(\widetilde{a}_{1}(s), \ldots, \widetilde{a}_{n}(s), \widetilde{g}_{1}(s), \ldots, \widetilde{g}_{m}(s)\right),
\end{aligned}
$$

whence (8) from Theorem 4 holds.

Step 4. In order to see that (6) and (8) determine $g_{1}, \ldots, g_{m}$, assume that $h_{1}, \ldots, h_{m} \in \mathcal{A}_{r}$ also satisfy these conditions. Then $c:=F\left(a_{1}, \ldots, a_{n}, h_{1}, \ldots, h_{n}\right)$ can be formed in $\mathcal{A}_{r}$. By (8), we have $\widetilde{c}(s)=\widehat{s}(c)=0$ for each $s \in \bar{H}_{r}$, whence $c=0$ and hence $\left(h_{1}, \ldots, h_{m}\right)=\left(g_{1}, \ldots, g_{m}\right)$, by uniqueness in Step 2 .

Remark 6. Suppose that $F: W \times Z \rightarrow \mathbb{C}^{m},\left(w_{0}, z_{0}\right) \in W \times Z$ and $a_{1}, \ldots, a_{n}$ are as in Theorem 4, but that $\left.\partial_{z} F\left(w_{0}, z\right)\right|_{z=z_{0}}$ fails to be invertible. Then a solution $\left(g_{1}, \ldots, g_{m}\right) \in \mathcal{A}^{m}$ to (6) and (7) can still be constructed provided that there exists 
a holomorphic function $\phi=\left(\phi_{1}, \ldots, \phi_{m}\right): W_{0} \rightarrow Z$ on an open neighbourhood $W_{0} \subseteq W$ of $w_{0}$ such that $\phi\left(w_{0}\right)=z_{0}$ and

$$
F(w, \phi(w))=0 \quad \text { for all } w \in W_{0} .
$$

In fact, the elements $g_{j}:=\phi_{j}\left(a_{1}, \ldots, a_{n}\right) \in \mathcal{A}$, defined using holomorphic functional calculus in $\mathcal{A}$, have the desired properties (this follows from (15) and the naturality of holomorphic functional calculus). If $a_{1}, \ldots, a_{n} \in \mathcal{A}_{\varrho}$ for some $\varrho \geq 0$, then Lemma 3(d) implies that there is an $r \geq \varrho$ such that $\sigma_{r}\left(a_{1}\right) \times \cdots \times \sigma_{r}\left(a_{n}\right) \subseteq$ $W_{0}$. Then $\phi_{j}\left(a_{1}, \ldots, a_{n}\right)$ can be formed in $\mathcal{A}_{r}$ and coincides with $g_{j}$, which yields $g_{1}, \ldots, g_{m} \in \mathcal{A}_{r}$. As above, we see that (7) holds in $\mathcal{A}_{r}$. Now applying the complex homomorphisms $\widehat{s}$ for $s \in \bar{H}_{r}$, we deduce that (8) is also satisfied.

\section{REFERENCES}

1. H. Biller, Analyticity and naturality of the multi-variable functional calculus, TU Darmstadt Preprint 2332, 2004; http://wwwbib.mathematik.tu-darmstadt.de/MathNet/Preprints/Listen/files/2332.ps.gz

2. T. Carroll and A.A. Gioia, Roots of multiplicative functions, Compositio Math. 65 (1988), 349-358. MR0932075 (89c:11007)

3. M.J. Cohen, Advanced problem 5293, Am. Math. Monthly 72 (1965), 555; Solution, ibid. 73 (1966), 553-554. MR1533818

4. S. Dierolf and J. Wengenroth, Inductive limits of topological algebras, Linear Topol. Spaces Complex Anal. 3 (1997), 45-49. MR.1632483 (2000c:46094)

5. P.-O. Dehaye, On the structure of the group of multiplicatice arithmetical functions, Bull. Belg. Math. Soc. 9 (2002), 15-21. MR1905645 (2003d:11009)

6. K. Floret, Lokalkonvexe Sequenzen mit kompakten Abbildungen, J. Reine Angew. Math. 247 (1971), 155-195. MR0287271(44:4478)

7. K. Floret and J. Wloka, Einführung in die Theorie der lokalkonvexen Räume, Springer-Verlag, Berlin, 1968. MR0226355 (37:1945)

8. H. Glöckner, Algebras whose groups of units are Lie groups, Studia Math. 153 (2002), 147177. MR.1948922(2003j:46066)

9. P. Haukkanen, Arithmetical equations involving semi-multiplicative functions and the Dirichlet convolution, Rend. Mat. Appl., VII. Ser. 8 (1988), 511-517. MR.1032719 (90k:11007)

10. P. Haukkanen, On the real powers of completely multiplicative arithmetical functions, Nieuw Arch. Wisk. 15 (1997), 73-77. MR1470439 (98h:11006)

11. E. Hewitt, and J.H. Williamson, Note on absolutely convergent Dirichlet series, Proc. Amer. Math. Soc. 8 (1957), 863-868. MR0090680(19:851b)

12. J. Knopfmacher, Abstract Analytic Number Theory, North-Holland, Amsterdam, 1975. MR0419383(54:7404)

13. V. Laohakosol, Divisors of some arithmetic functions, in: Tangmanee, E. (ed.) et al., Proceedings of the second Asian mathematical conference 1995, Nakhon Ratchasima, Thailand, October 17-20, 1995. World Sci. Publishing, River Edge, NJ, 1998, 139-151. MR1660560 (2001f:11008)

14. S. Porubský, Structure of the group of quasi multiplicative arithmetical functions, Acta Arith. Paed. Agiensis, Sect. Math. 30 (2003), 133-145. MR2054723 (2005b:11006)

15. D. Rearick, Divisibility of arithmetic functions, Pacific J. Math. 112 (1984), 237-248. MR:0739148 (85j:11011)

16. W. Schwarz, Ramanujan-Entwicklungen stark multiplikativer zahlentheoretischer Funktionen, Acta Arith. 22 (1973), 329-338. MR0323740 (48:2096)

17. M.V. Subbarao, A class of arithmetical functions, Nieuw Archief voor Wiskunde (3) 15 (1967), 211-217. MR0223293 (36:6341)

18. L. Waelbroeck, Les algèbres à inverse continu, C.R. Acad. Sci. Paris 238 (1954), 640-641. MR0073951 (17:513a) 
Fachbereich Mathematik, TU Darmstadt, Schlossgartenstrasse 7, 64289 Darmstadt, GERMANY

E-mail address: gloeckner@mathematik.tu-darmstadt.de

Institute of Mathematics, Clausthal University of Technology, Erzstrasse 1, 38678 Clausthal-Zellerfeld, Germany

E-mail address: lucht@math.tu-clausthal.de

Institute of Computer Science, Academy of Sciences of the Czech Republic, Pod Vodárenskou věží 2, 18207 Prague 8, Czech Republic

E-mail address: Stefan.Porubsky@cs.cas.cz 